\title{
POSITRON EMISSION TOMOGRAPHY WITH F18-FLUORODEOXYGLUCOSE IN THE STAGING AND PREOPERATIVE EVALUATION OF MALIGNANT PLEURAL MESOTHELIOMA
}

Darren B. Schneider, MD

Carolyn Clary-Macy, RN

Sudha Challa, MD

Kent C. Sasse, MD

Scott H. Merrick, MD

Randall Hawkins, MD

Gary Caputo, MD

David Jablons, MD
Objectives: The purpose of this study was to evaluate the utility of positron emission tomography with F18-fluorodeoxyglucose in the preoperative evaluation and staging of malignant mesothelioma in patients who were candidates for aggressive combined modality therapy.

Methods: Eighteen consecutive patients with biopsy-proven malignant mesothelioma underwent positron emission tomographic scanning. The results of positron emission tomographic imaging were compared with results obtained by computed tomography, mediastinoscopy, thoracoscopy, and pathologic examination of surgical specimens. All patients fasted and received an average of $14.5 \pm 2.7 \mathrm{mCi}$ of F18-fluorodeoxyglucose for positron emission tomographic scanning. Attenuation-corrected whole-body and regional emission images of the chest and upper abdomen were acquired and formatted into transaxial, coronal, and sagittal images.

Results: All primary malignant mesotheliomas accumulated F18-fluorodeoxyglucose, and the mean standardized uptake value was 7.6 (range, 3.33$14.85 ; n=9)$. There were no false-negative results of positron emission tomography. Identification of occult extrathoracic metastases by positron emission tomography was the basis for excluding two patients from surgical therapy. There were two false-positive results of positron emission tomography: increased F18-fluorodeoxyglucose uptake in the contralateral chest that was negative by thoracoscopic biopsy $(n=1)$ and increased abdominal F18-fluorodeoxyglucose uptake after partial colectomy for diverticular disease $(n=1)$.

Conclusions: Positron emission tomography can identify malignant pleural mesothelioma and appears to be a useful noninvasive staging modality for patients being considered for aggressive combined modality therapy. ( $\mathrm{J}$ Thorac Cardiovasc Surg 2000;120:128-33)
$\mathrm{M}^{\mathrm{a}}$ alignant pleural mesothelioma is uncommon in the United States, but its incidence is rising. Because of patterns of occupational asbestos exposure, the annual incidence of 3000 new cases is expected to increase by over $50 \%$ in the coming decade. ${ }^{1,2}$ Without

From the Division of Cardiothoracic Surgery and the Departments of Surgery and Nuclear Medicine, University of California/Mount Zion Medical Center, San Francisco, Calif.

Read at the Twenty-fifth Annual Meeting of The Western Thoracic Surgical Association, Olympic Valley (Lake Tahoe), Calif, June 23-26, 1999.

Received for publication June 29, 1999; revisions requested Aug 30, 1999; revisions received Jan 28, 2000; accepted for publication Feb 14, 2000.

Address for reprints: David Jablons, MD, Division of Thoracic Surgery, Mount Zion-UCSF Cancer Center, 1600 Divisadero St, Rm C322, San Francisco, CA 94143-1674.

Copyright (c) 2000 by The American Association for Thoracic Surgery

$0022-5223 / 2000 \$ 12.00+0 \quad \mathbf{1 2 / 6 / 1 0 6 5 2 9}$

doi: $10.1067 / \mathrm{mtc} .2000 .106529$ therapy, survival is dismal and rarely exceeds 12 months. Recently, however, several reports suggest that aggressive combined modality therapy, including either radical pleurectomy or extrapleural pneumonectomy, followed by chemotherapy and radiation, may significantly prolong survival., 3

Improvements in the detection of regional and distant metastases are needed to identify patients most likely to benefit from aggressive combined-modality treatment regimens. Notably, most long-term survivors after combined modality treatment have disease limited to the pleural space without mediastinal lymph node or distant metastases. ${ }^{4,5}$ Although computed tomography (CT) and magnetic resonance imaging (MRI) are routinely used to predict resectability, ${ }^{6}$ these imaging modalities fail to accurately demonstrate chest wall or transdiaphragmatic invasion and have a poor sensitivity for detecting mediastinal lymph node involvement. ${ }^{7,8}$ Morphometric analysis of primary tumor volume may 
predict survival on the basis of $\mathrm{T}$ status, but it is a difficult and time-consuming technique that analyzes only the local extent of disease. ${ }^{9}$

Uptake of the positron-emitting glucose analog F18fluorodeoxyglucose (FDG) is increased in metabolically active malignant cells and is the basis for its widespread use in positron emission tomography (PET) imaging. FDG-PET is proving increasingly valuable in the preoperative staging of lung and other cancers. ${ }^{10-13}$ Because PET reflects the metabolic activity of tissues, PET is complementary to anatomic imaging studies, such as CT or MRI. Although the precise role of PET in cancer treatment algorithms is yet to be determined, multiple studies support the use of PET in the workup of patients with lung, breast, colorectal, and head and neck cancers. ${ }^{14}$ Recently, Bénard and colleagues ${ }^{7}$ reported the ability of PET to differentiate malignant mesothelioma from benign pleura-based disease and suggested that it may also be useful in preoperative staging. To evaluate the utility of PET in the preoperative evaluation and staging of malignant mesothelioma, we reviewed data collected from a consecutive series of patients referred for treatment of malignant mesothelioma. We hypothesized that PET could improve detection of mediastinal lymph nodes and distant metastatic disease, thereby facilitating the selection of patients who would benefit from aggressive combined modality treatment.

\section{Methods}

The records from 18 consecutive patients referred for treatment of malignant pleural mesothelioma who underwent FDG-PET studies between 1997 and 1999 were reviewed. All patients had a diagnosis of mesothelioma established by means of open or thoracoscopic pleural biopsy or by cytologic examination of pleural fluid. Pathologic slides from other hospitals were reviewed by our pathology department to confirm the diagnosis. All patients underwent a comprehensive evaluation, which included history and physical examination, chest radiography, pulmonary function testing, CT of the chest and upper abdomen, and PET scans. Additional diagnostic procedures, such as thoracoscopy, laparoscopy, and mediastinoscopy, were obtained when clinically indicated.

Eight of the PET scans were obtained at the University of California San Francisco PET imaging center, and the other 10 scans were conducted at a total of 4 other centers. Similar imaging protocols were used at each center. All patients were asked to fast for at least 4 hours before PET scanning. Normoglycemia was confirmed by measurement of capillary blood glucose levels. Approximately 40 minutes after the intravenous administration of $14.5 \pm 2.7 \mathrm{mCi}$ of FDG, patients were placed in the supine position in the PET scanner (Siemens ECAT; Siemens-Elema, Division of ElemaSchönander, Inc, Solna, Sweden). Separate attenuation-cor- rected transmission and emission scans of the thorax and upper abdomen were acquired in two bed positions. Whole body emission scans from the base of the skull to the thighs were acquired in 6 bed positions. Images were reconstructed in the transaxial, coronal, and sagittal planes.

PET scans were interpreted independently by an experienced nuclear medicine physician. CT reports were available for comparison. PET images were considered to be positive for malignancy if there was focal hypermetabolic activity with FDG uptake greater than background or mediastinal activity. Standardized uptake value (SUV) was available for the last 9 patients in the series and was calculated as described. ${ }^{15}$ PET scan reports also included the likelihood of malignancy in the contralateral chest, mediastinal lymph nodes, or extrathoracic sites. Mediastinal lymph nodes were defined as positive if discrete mediastinal foci of FDG uptake not located within the pleural reflection could be identified.

Patient management decisions were based on the combined interpretation of CT and PET images. Patients with suspected metastatic disease involving the contralateral chest, mediastinal lymph nodes, or extrathoracic sites underwent confirmatory diagnostic procedures (including thoracoscopy of the contralateral chest, mediastinoscopy, laparoscopy, or open incisional biopsy). Patients found to have disease limited to the pleural space without metastases underwent aggressive combined modality therapy consisting of radical pleurectomy and decortication, mediastinal lymph node sampling, and intraoperative radiotherapy followed by postoperative chemotherapy and radiation. Complete mediastinal lymph node dissections were not routinely performed. All PET and CT scans were reviewed in conjunction with pathology reports from biopsy, surgical specimens, or autopsy reports.

\section{Results}

A total of 18 consecutive patients with pleural mesothelioma were evaluated with PET scanning. The median age was 68 years (range, $43-83$ years), and the male/female ratio was 14:4. A history of asbestos exposure was documented in $13(72 \%)$ patients, and 14 (78\%) patients reported a history of tobacco use. The mesothelioma cell type was epithelial or mixed in 17 (94\%) patients and was sarcomatous in $1(6 \%)$ patient. Eleven $(61 \%)$ patients had mesothelioma of the right side of the chest, and 7 (39\%) patients had tumors of the left side of the chest. Ten (56\%) patients had undergone talc pleurodesis for the treatment of effusions associated with malignant mesothelioma (ranging from 1 to 24 months before PET scanning). Twelve (67\%) patients were treated with aggressive combined modality treatment consisting of radical pleurectomy and decortication with intraoperative radiotherapy followed by postoperative chemotherapy and radiation. In 2 (11\%) patients radical pleurectomy was attempted but was aborted because of extensive chest wall invasion. 

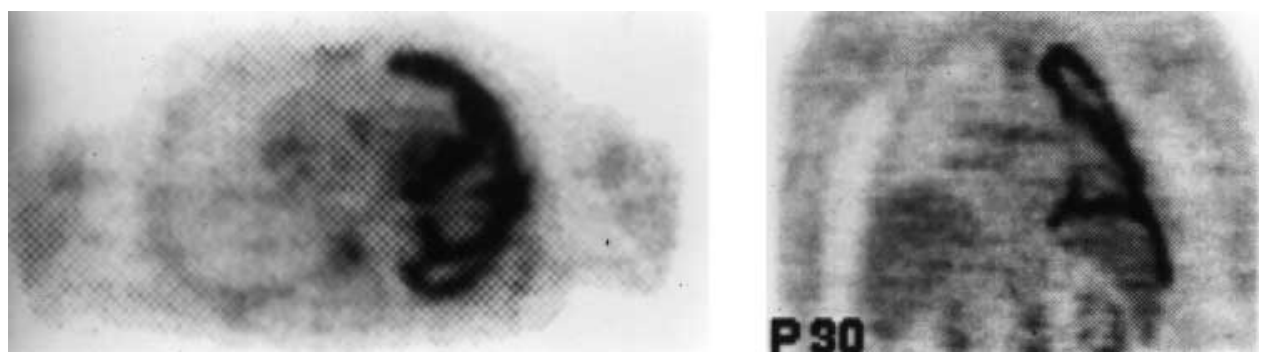

Fig 1. Transverse (left) and coronal (right) PET images demonstrating extensive involvement of pleural surfaces, including interlobar fissures and diaphragmatic surfaces, by mesothelioma.
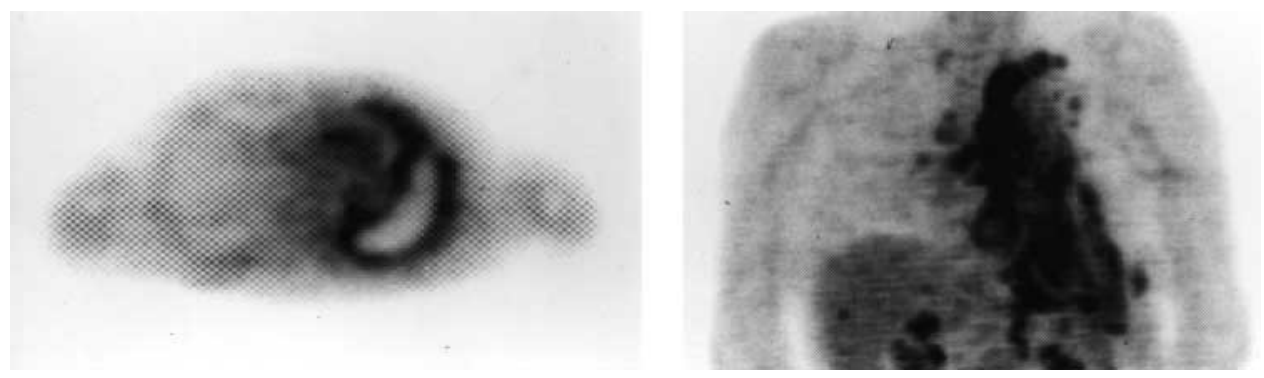

Fig 2. Left, Transverse PET image displaying increased focus of FDG uptake by mediastinal lymph nodes. Right, Coronal section of a patient with extensive metastases to mediastinal, porta hepatis, and celiac lymph nodes.

Four $(22 \%)$ patients who were found to have metastases to mediastinal lymph nodes or extrathoracic sites did not undergo radical pleurectomy.

All 18 primary mesotheliomas accumulated significant amounts of FDG, and the mean SUV of these tumors was $7.5 \pm 4.2$. The pattern of FDG uptake typically matched the CT and intraoperative findings with regard to the extent of pleural involvement and tumor burden. Involvement of the interlobar fissures, as well as the diaphragmatic surfaces, was also clearly demonstrated by PET (Fig 1). The presence of pleural effusion did not interfere with FDG uptake. However, PET failed to predict transdiaphragmatic involvement or chest wall invasion in 2 patients.

PET scanning identified increased FDG uptake in mediastinal lymph nodes in 4 patients (Fig 2). In all 4 subjects lymph node metastases were confirmed by pathologic examination of either biopsy or surgical specimens. PET did not demonstrate lymph node involvement in the remaining $14(78 \%)$ patients, and absence of lymph node involvement was confirmed by pathologic examination in 12 of these 14 cases. In contrast, CT demonstrated lymph node enlargement in only 2 of the 4 subjects with documented lymph node metastases. In 2 patients $\mathrm{CT}$ gave false-positive results by detecting enlarged lymph nodes, which were benign by pathologic examination.

Overall, PET correctly identified the presence or absence of metastatic disease in $16(89 \%)$ of the 18 patients. Identification of occult extrathoracic metastases, including axillary and cervical lymph node metastases and bone metastases, by PET was the basis for excluding 2 patients from surgical therapy. PET also confirmed the CT finding of a contralateral lung metastasis in 1 patient. In 2 patients negative PET scans also confirmed that contralateral pleural abnormalities identified by $\mathrm{CT}$ were benign. In 2 patients increased FDG uptake by benign processes resulted in false-positive PET scans. In 1 case increased pleural uptake in the contralateral chest was falsely interpreted as metastatic disease. Notably, the intensity of FDG uptake was less than the uptake by the patient's primary tumor, and thoracoscopy with multiple biopsies was consistent with a benign asbestos-related plaque. Another patient who had undergone a partial colectomy for diverticulitis 2 months previously had an abdominal focus of increased FDG uptake that represented residual hypermetabolic activity.

The accuracy of the PET scans in detecting metastatic disease in mediastinal lymph nodes or distant sites 
is summarized in Table I. Two patients with extensive chest wall involvement could not undergo radical pleurectomy and did not have mediastinal lymph nodes sampled. The remaining 16 patients had histologic examination of mediastinal lymph nodes or distant metastases confirmed. Of these 16 patients, PET correctly predicted the presence or absence of metastatic disease in 14 patients. In contrast, CT correctly predicted the presence or absence of metastatic disease in only 8 of 16 patients.

\section{Discussion}

Our findings suggest that PET is a useful imaging modality for the preoperative staging of malignant mesothelioma. These results are consistent with findings recently reported by Bénard and colleagues, ${ }^{7}$ who found that PET had a sensitivity and specificity of $91 \%$ and $100 \%$, respectively, for the identification of primary mesotheliomas. They found that PET results were positive for lymph node metastases in 12 of 24 patients with mesothelioma, but only 10 of 24 patients had confirmation by surgical staging. Nonetheless, in the subjects with pathologic confirmation, they reported a sensitivity and specificity of $83 \%$ and $75 \%$ of PET for lymph node metastases. Our results also suggest that PET may identify involvement of mediastinal lymph nodes or extrathoracic sites that are underappreciated by CT.

It is a common but incorrect belief that mesothelioma tends to progress locally, and lymph node or distant metastases may rarely occur. Although mesothelioma tends to progress locally rather than systemically, lymph node metastases may be present more often than was formerly believed. In addition, several recently published trials of aggressive combined modality treatment have emphasized the adverse effect of nodal metastases on survival. ${ }^{4,5}$ Rusch and Venkatraman ${ }^{4}$ reported that among 89 patients who underwent lymph node dissections, 50\% had metastases to N2 mediastinal nodes. Sugarbaker and colleagues ${ }^{5}$ found that positive extrapleural lymph node status significantly shortened survival after extrapleural pneumonectomy followed by chemotherapy and radiation. Accordingly, staging systems recently proposed by both the International Mesothelioma Interest Group ${ }^{16}$ and Sugarbaker's group ${ }^{5}$ place greater importance on nodal status than the system previously described by Butchart and colleagues. ${ }^{17}$ In this study we classified only discrete, nonadjacent mediastinal foci of FDG uptake as a positive result indicating nodal disease. This is because the poor spatial resolution of PET combined with the
Table I. Efficacy of PET and CT scans in detecting mediastinal lymph nodes or distant metastatic disease

\begin{tabular}{lcc}
\hline & $C T$ & $P E T$ \\
\hline True positive & 3 & 5 \\
True negative & 5 & 9 \\
False positive & 4 & 2 \\
False negative & 4 & 0 \\
\hline
\end{tabular}

Analysis includes 16 patients who had pathologic examination of mediastinal lymph nodes $(n=14)$ or distant metastases confirmed by other methods (biopsy or $\mathrm{CT}, \mathrm{n}=2$ ). A patient was considered to have metastatic disease if involvement of mediastinal lymph nodes, distant sites, or both was identified.

intense glucose uptake by the primary tumor can make it difficult to distinguish between involved N1 (intrapleural nodes) and the primary tumor. This definition is supported by Sugarbaker's staging system, ${ }^{5}$ wherein extrapleural, as opposed to intrapleural, nodal involvement is specifically associated with an adverse prognosis. Our data suggest that by detecting extrapleural nodal disease, PET may improve patient selection for combined modality treatment.

When compared with CT as a staging modality for malignant mesothelioma, PET has both advantages and disadvantages. In our experience, PET does not accurately predict the presence or extent of local invasion into the chest wall, pericardium, or diaphragm. The greater anatomic detail afforded by CT and MRI is more useful for imaging the local extent of disease and predicting resectability. Indeed, the sensitivity of CT and MRI for predicting resectability exceeds $90 \%{ }^{6}$ PET, however, provides complementary information by distinguishing benign from malignant pleura-based disease. ${ }^{7}$ Because PET identifies tissues with increased glucose uptake, false-positive results may occur at sites of inflammation. ${ }^{18}$ However, only 2 false-positive results were identified by PET in our series compared with the 4 false-positive results obtained with CT (Table I). Importantly, PET identified undetected distant metastases in 2 patients, preventing aggressive treatment that was unlikely to improve their survival. Nonetheless, PET scan findings must still be correlated with CT findings and the clinical picture. When discrepancies occur and the PET scan does not fit the clinical picture, surgical staging (eg, mediastinoscopy, thoracoscopy, and open biopsy) remains definitive and necessary.

Although our data support the use of PET as a staging modality for malignant mesothelioma, the best method to analyze the accuracy of PET would be a comparison of PET, CT, and pathologic analysis in prospective fashion. Our study is limited by its retro- 
spective design, small sample size, and the absence of mediastinal lymph node sampling in every patient. Without complete mediastinal lymph node dissection, including internal mammary and peridiaphragmatic nodes, in each patient, it is impossible to determine the exact sensitivity and specificity of PET for detecting metastatic disease. Moreover, approximately one half of our patients underwent talc pleurodesis before PET scanning, which may have falsely elevated the amount of FDG uptake. Further studies are warranted to assess the utility of PET and other staging modalities, such as mediastinoscopy, in the staging of mesothelioma.

In conclusion, PET appears to be a useful imaging modality for the evaluation and staging of malignant mesothelioma. Because PET images tumors on the basis of their biologic behavior, it is particularly valuable for distinguishing between benign and malignant pleural processes. Moreover, PET may also allow noninvasive staging of mediastinal nodal status and extrathoracic metastases. False-positive results may occasionally be produced by nonmalignant processes; however, a negative PET scan result provides strong evidence that the lesion is benign. On the basis of our findings, we routinely obtain both CT and PET scans on all patients referred for the evaluation of mesothelioma. By identifying the metastatic spread of disease preoperatively, we believe that PET may improve the selection of patients who are most likely to benefit from aggressive multimodality therapy for mesothelioma.

\section{REFERENCES}

1. Walker AM, Loughlin JE, Friedlander ER, Rothman KJ, Dreyer NA. Projections of asbestos-related disease 1980-2009. J Occup Med 1983;25:409-25.

2. Connelly RR, Spirtas R, Myers MH, Percy CL, Fraumeni JF Jr. Demographic patterns for mesothelioma in the United States. J Natl Cancer Inst 1987;78:1053-60.

3. Rice TW, Adelstein DJ, Kirby TJ, Saltarelli MG, Murthy SR, Van Kirk MA, et al. Aggressive multimodality therapy for malignant pleural mesothelioma. Ann Thorac Surg 1994;58:24-9.

4. Rusch VW, Venkatraman E. The importance of surgical staging in the treatment of malignant pleural mesothelioma. J Thorac Cardiovasc Surg 1996;111:815-26.

5. Sugarbaker DJ, Flores RM, Jaklitsch MT, Richards WG, Strauss GM, Corson JM, et al. Resection margins, extrapleural nodal status, and cell type determine postoperative long-term survival in trimodality therapy of malignant pleural mesothelioma: results in 183 patients. J Thorac Cardiovasc Surg 1999;117:54-65.

6. Patz EF Jr, Shaffer K, Piwnica-Worms DR, Jochelson M, Sarin M, Sugarbaker DJ, et al. Malignant pleural mesothelioma: value of CT and MR imaging in predicting resectability. AJR Am J Roentgenol 1992;159:961-6.

7. Bénard F, Sterman D, Smith RJ, Kaiser LR, Albelda SM, Alavi A. Metabolic imaging of malignant pleural mesothelioma with fluo- rodeoxyglucose positron emission tomography. Chest 1998;114:713-22.

8. Rusch VW, Godwin JD, Shuman WP. The role of computed tomography scanning in the initial assessment and the follow-up of malignant pleural mesothelioma. J Thorac Cardiovasc Surg 1988;96:171-7.

9. Pass HI, Temeck BK, Kranda K, Steinberg SM, Feuerstein IR. Preoperative tumor volume is associated with outcome in malignant pleural mesothelioma. J Thorac Cardiovasc Surg 1998;115:310-8.

10. Block MI, Patterson GA, Sundaresan RS, Bailey MS, Flanagan FL, Dehdashti F, et al. Improvement in staging of esophageal cancer with the addition of positron emission tomography. Ann Thorac Surg 1997;64:770-7.

11. Graeber GM, Gupta NC, Murray GF. Positron emission tomographic imaging with fluorodeoxyglucose is efficacious in evaluating malignant pulmonary disease. J Thorac Cardiovasc Surg 1999;117:719-27.

12. Lowe VJ, Naunheim KS. Positron emission tomography in lung cancer. Ann Thorac Surg 1998;65:1821-9.

13. Blend MJ, Abdel-Nabi H. New methods for the staging of colorectal cancer using noninvasive techniques. Semin Surg Oncol 1996;12:253-63.

14. Silverman DH, Hoh CK, Seltzer MA, Schiepers C, Cuan GS, Gambhir SS, et al. Evaluating tumor biology and oncological disease with positron-emission tomography. Semin Radiat Oncol 1998;8:183-96.

15. Zasadny KR, Wahl RL. Standardized uptake values of normal tissues at PET with 2-[fluorine-18]-fluoro-2-deoxy-D-glucose: variations with body weight and a method for correction. Radiology 1993;189:847-50.

16. Rusch VW. A proposed new international TNM staging system for malignant pleural mesothelioma. From the International Mesothelioma Interest Group. Chest 1995;108:1122-8.

17. Butchart EG, Ashcroft T, Barnsley WC, Holden MP. Pleuropneumonectomy in the management of diffuse malignant mesothelioma of the pleura: experience with 29 patients. Thorax 1976;31:15-24.

18. Patz EF Jr, Lowe VJ, Hoffman JM, Paine SS, Burrowes P, Coleman RE, et al. Focal pulmonary abnormalities: evaluation with F-18 fluorodeoxyglucose PET scanning. Radiology 1993;188:487-90.

\section{Discussion}

Dr Eric Vallieres (Seattle, Wash). You and your colleagues have shown us another potentially powerful use of PET in the workup and staging of a thoracic malignancy. I am also fortunate to have on-site access to this technology, and I appreciate almost on a daily basis the additional diagnostic staging and prognostic information the test provides me and my cancer patients. PET scanning is now recognized as a staging tool for lung cancer, lymphoma, and a growing number of other malignant diseases. Universal access to PET scanning remains an issue, however, but as its value becomes recognized, this limitation should disappear. Reports like this one are needed, and they will serve to establish the place of this test in the evaluation of patients with mesothelioma. The group at the University of Pennsylvania, as you have cited, has already indicated how useful PET may possibly be in dif- 
ferentiating benign and malignant pleural changes, a sometimes very difficult differentiation to make in asbestosexposed patients who have pleural changes by conventional imaging.

In this series of 18 patients with a proven diagnosis of mesothelioma, PET scanning is shown to be a powerful mediastinal staging noninvasive tool with a reported sensitivity of $100 \%$ in the 16 patients in whom pathologic correlation was available. This compares with a $43 \%$ sensitivity for CT scan. We know that the presence of N2 disease in mesothelioma carries a bad prognosis that is probably not altered by therapy. Hence, it is important to select out these patients before submitting them to potentially very morbid and costly multimodality therapy. The same argument applies to the identification of unsuspected systemic metastasis in these patients, $2(11 \%)$ of 18 in your series, sparing a probably futile aggressive therapy in view of the PET findings. The numbers are small but compare with the range of $17 \%$ to $20 \%$ of patients with potentially resectable lung cancer in whom unsuspected M1 disease is identified by means of PET scanning. It is a great test, but one has to be careful, and as shown in this report, a positive PET does not always indicate cancer. False positivity is low but exists, and we have to be careful not to write off patients with a chance at cure without histologic confirmation of the suspected findings.

Dr Schneider, I have 3 questions regarding your presentation. We know from the growing lung cancer PET literature that emission scans may underestimate the activity in mediastinal lymph nodes, particularly when the tumor is central or paramediastinal, such as that found with mesothelioma. These nodes, however, can be seen and better evaluated by attenuation-corrected transmission scans. Were attenuationcorrected transmission scans of the mediastinal area obtained in all patients? If so, why was an SUV available in only 6 of your patients?

Dr Schneider. Thank you for your comments. With regard to your first question, we did obtain attenuation-corrected transmission images in all of our patients. However, a num- ber of these PET scans were not performed at UCSF, and we are actually in the process of gathering all those data and reviewing the SUVs on the entirety of our series right now.

Dr Vallieres. More than half of your patients had received a talc pleurodesis before the PET evaluation. It is known that inflammatory changes are usually "hot" on PET, and the patient with a healing colonic anastomosis proved that point in your series. How early after this pleurodesis were the PET scans obtained, and do you think that the inflammation of pleurodesis may have somehow influenced the data presented, particularly in regard to the mean SUV reported in your document?

Dr Schneider. It is entirely possible that talc pleurodesis did affect some of the SUVs that were obtained. Some of the talc pleurodeses were as little as 3 or 4 weeks before the PET scanning. Nonetheless, all the tumors did have increased FDG uptake. We particularly wanted to evaluate areas outside of the area where the talc was applied, such as the mediastinum and distant sites.

Dr Vallieres. Finally, your numbers remain small, but this is the largest series on the topic to date, and a sensitivity of $100 \%$ is to be noted. In your opinion, what is the role of mediastinoscopy in staging the mediastinum in a potential candidate for multimodality therapy in this era of PET scanning?

Dr Schneider. We have recently started doing mediastinoscopy in some of our patients with mesothelioma, in particular those who have positive PET scan results, just to confirm that those lymph nodes are indeed positive and not a false-positive result. We have been routinely performing mediastinoscopy in patients with positive PET scan results for mediastinal nodes.

Dr Vallieres. If the PET is "cold," you do not?

Dr Schneider. We have to combine the PET results with all the different imaging modalities and the clinical picture as well. Therefore, if the PET does not fit the picture, then we use these other tests such as mediastinoscopy. We have not come up with a formal protocol, but we are using mediastinoscopy more frequently. 